\title{
Looking for the Integrome, When Metabolomics Talk about the Interactome
}

\author{
Juan Bueno* \\ Research Center of Bioprospecting and Biotechnology for Biodiversity Foundation (BIOLABB), Colombia
}

\begin{abstract}
Secondary metabolites are not produced in isolation; they are the result of the interaction of genes, metabolism and the environment. These interaction networks that are part of the biological systems are the most reliable expression of the functioning of living beings and is a great tool to obtain information that leads to promising applications in the diagnosis of diseases, drug development, as well as the conservation and restoration of ecosystems. In this way the multi-omics approach which constitutes the confluence of various high-throughput technologies that seeks to integrate all platforms in a multivariate model that provide comprehensive profiles of the same phenomenon, it confers the ability to be the link between different aspects of cellular populations relevant in biotechnology and ecosystems research. In this order of ideas, the aim of this editorial is to give an unifying canon under the concept of integrome, like that search result of the fusion of several omics disciplines that seek to solve problems by applying the discoveries.
\end{abstract}

Keywords: Integrome; Metabolomics; Interactome; Multi-omics approach

\section{Introduction}

Integrome is defined as the connection networks that describe the interaction, the construction of this intercommunication system is the product of a multivariate analysis of the data obtained by the combination of several omics technologies (genomics, transcriptomics, metabolomic and proteomics) [1]. In this way, experimental omics approaches, share different characteristics as are: to be high-throughput and massive data-based; study the molecular phenomena, as an "integrated system" due to the incorporation of the interrelation between the information obtained; and to require a complex multivariate statistical analysis because to the large amount of data [2]. This multi-trans-inter-omics needs combine several methodologies with a previous hypothesis as are genome sequencing, RNA sequencing, chromatin immunoprecipitation sequencing (ChIP-seq), mass spectrometry (MS), expression proteomics, liquid chromatography, capillary electrophoresis and nuclear magnetic resonance (NMR) [3]. Following this order of ideas an integrated-omics conceptualization is necessary in microbiology looking for detect different properties of microbial cells in multiple functional states, with the objective of reduce the information disparity between gene expression and phenotypic variation [4]. Also, is very important analyze using bioinformatics tools of the entire omics dataset for determine the genetic associations (genome-wide association studies) and their interactions with metabolites as well as protein expression, with the end to elucidate the biochemical pathways and molecular targets the most used statistical instruments are BioGrid, JActiveModules, BioNet, DEGAS, GiGa, HotNet, NuChart and Key Pathway Miner Web [5-7]. In addition, epigenome association studies is other interesting factor for to take in account looking for to establish the effect of the environment on the cellular response [8]. In short, the correct elaboration of the working hypothesis is what defines the problem that requires the use of the integrome [9], because discoveries need questions before everything.

\section{Epigenetics the discovery of the unified field}

In this way seeking for a multi-omics approach that can be included in a translational research concept, it will be very important perform environmental epigenetics studies that can provides explanations about the molecular mechanisms that directly cause phenotypic changes [10], this neo-Lamarckian concept have to be unified for to perform a holistic approach that describes an autopoietic model in which is possible to establish the physical and chemical processes through which the system under study exists [11]. In addition, it is important to remember that this system as unity must comply with the rule of self-regenerating itself continuously as well as its network of processes [11]. So that, a real multi-omics research work must move towards a molecular autopoiesis thought describing the operation of unicellular and multicellular living beings [12].

\section{Multi-omics age}

In fact multi-omics research is an integrative platform which can be used in combination with clinical data for the diagnosis of complex diseases [13], as well as being applied to determine the multi-target activity of drugs with which is possible to discover new treatments and predict drug interactions based on intercellular networks. In addition, it can determine what medications could be used in combination therapy for treatments resistant to conventional medication [14]. In this order of ideas, the functionality of cell populations will be a big step in this area, developing promising tools such as metatranscriptomic, metaproteomic or metametabolomic [15]. This new passion for integration will be able to evaluate our connection as living beings with our environment through our biological evolution from primal organisms to organized microbiomes [16]. This is how biological research accesses an ecosystem approach that will define us as symbiotic organisms beyond the isolated study of processes.

*Corresponding author: Juan B, Research Center of Bioprospecting and Biotechnology for Biodiversity Foundation (BIOLABB), Colombia, E-mail: juanbueno@biolabb.com; juangbueno@gmail.com

Received December 26, 2017; Accepted January 18, 2018; Published January 25,2018

Citation: Bueno J (2018) Looking for the Integrome, When Metabolomics Talk about the Interactome. J Microb Biochem Technol 10: e134. doi:10.4172/19485948.1000e134

Copyright: (c) 2018 Bueno J. This is an open-access article distributed under the terms of the Creative Commons Attribution License, which permits unrestricted use, distribution, and reproduction in any medium, provided the original author and source are credited. 
Citation: Bueno J (2018) Looking for the Integrome, When Metabolomics Talk about the Interactome. J Microb Biochem Technol 10 : e134. doi:10.4172/1948-5948.1000e134

\section{References}

1. Ohashi H, Hasegawa M, Wakimoto K, Miyamoto-Sato E (2015) Next-generation technologies for multiomics approaches including interactome sequencing. Biomed Res Int 2015:104209.

2. Huang H, Vangay P, McKinlay CE, Knights D (2014) Multi-omics analysis of inflammatory bowel disease. Immunol Lett 162: 62-68.

3. Yugi K, Kubota H, Hatano A, Kuroda S (2016) Trans-omics: How to reconstruct biochemical networks across multiple 'omics' layers. Trends Biotechnol 34 276-290.

4. Fondi, M, Liò $P$ (2015) Multi-omics and metabolic modelling pipelines: Challenges and tools for systems microbiology. Microbiol Res 171: 52-64.

5. Dumas ME (2012) Metabolome 2.0: Quantitative genetics and network biology of metabolic phenotypes. Mol Biosyst 8: 2494-2502.

6. Merelli I, Liò P, Milanesi L (2013) NuChart: An R package to study gene spatial neighbourhoods with multi-omics annotations. PLoS One 8: e75146.

7. List M, Alcaraz N, Dissing-Hansen M, Ditzel H J, Mollenhauer J, et al. (2016) Key pathway miner web: Online multi-omics network enrichment. Nucleic Acids Res 44: W98-W104.

8. Birney E, Smith GD, Greally JM (2016) Epigenome-wide association studies and the interpretation of disease-omics. PLoS Genet 12: e1006105.
9. Baker M (2013) The'omes puzzle. Nature 494: 416.

10. Skinner MK (2015) Environmental epigenetics and a unified theory of the molecular aspects of evolution: A neo-Lamarckian concept that facilitates neoDarwinian evolution. Genome Biol Evol 7: 1296-1302.

11. Nomura T (2003) Formal description of autopoiesis for analytic models of life and social systems. Proceedings of the Eighth International Conference on Artificial Life 1: 15-18.

12. Razeto-Barry $P$ (2012) Autopoiesis 40 years later. A review and a reformulation Orig Life Evol Biosph 42: 543-567.

13. Higdon R, Earl RK, Stanberry L, Hudac CM, Montague E, et al (2015) The promise of multi-omics and clinical data integration to identify and target personalized healthcare approaches in autism spectrum disorders. Omics 19:197-208.

14. Karaosmanoglu K, Sayar NA, Kurnaz IA, Akbulut BS (2014) Assessment of Berberine as a multi-target antimicrobial: A multi-omics study for drug discovery and repositioning. Omics 18: 42-53.

15. Aguiar-Pulido V, Huang W, Suarez-Ulloa V, Cickovski T, Mathee K, et al (2016) Metagenomics, metatranscriptomics, and metabolomics approaches for microbiome analysis. Evol Bioinform Online 12: 5-16.

16. Pérez-Cobas AE, Gosalbes MJ, Friedrichs A, Knecht H, Artacho A, et al (2012) Gut microbiota disturbance during antibiotic therapy: A multi-omic approach. Gut 62: 1591-1601. 